\title{
Hierarchy Structure of Root's Plant: The Part-Whole Relation in Corpus Linguistics
}

\author{
Rizka Fahrina Daulay ${ }^{[1]}$ and Totok Suhardijanto \\ \{1rizka.fahrina@ui.ac.id, totok.suhardijanto@ui.ac.id\} \\ ${ }^{1}$ Linguistics Program, Faculty of Humanities, Universitas Indonesia
}

\begin{abstract}
This study deals with lexical semantics field of hierarchical partwhole relation of root's plant lexeme, using a corpus linguistic approach to screen the data and statistically find out the frequency of co-occurrence of each root-related lexeme and its collocations. The analysis was carried out by employing Cruse's notion of lexical configuration to build hierarchical levels. Additionally, a concordance analysis was also employed to determine the meronymy among each lexeme that is part of the root. In Wikipedia Corpus, three dominant elements of plant are found, namely root, stem, and leaf. However, for the purpose of conclusiveness, this study only focuses on root, particularly the collocations of each of its parts as seen in Wikipedia Corpus. This study is expected to contribute to references in lexical semantics, especially in determining the paradigmatic relation of hierarchical formation in a concrete and structured manner, by employing corpus linguistics and taking account of scholar descriptions.
\end{abstract}

Keywords: root anatomy, meronymy, collocation, concordance

\section{Introduction}

The notion of sintagmatic and paradigmatic relations has been widely known in studies of structural linguistics. This study, however, focuses on paradigmatic relation in particular. According to Murphy [1], words that have a paradigmatic relation have the same part of speech and characteristics. In a paradigmatic relation, words are closely linked in a way that can be seen from the relationships between the lexemes, one of which is expressed as a partwhole relation. Meronymy, on the other hand, is an asymmetric relation, as, for instance, seen in stanza and poem, with stanza being a meronym of poem and poem being a holonym of stanza [2].

Cruse [3] already mention about transitivity of the part-whole relation and this view is affirmed by Cruse [4], Croft \& Cruse [5] that state that meronymy is a relation between meaning, in which the part-whole relation connects two individual entities. This relation between meronym and holonym form a hierarchy. Meronymy is a part/portion-whole relation applied to two individual entities to illustrate the notion of containment, as, for example, seen in finger-hand. Using this finger-hand pair as the example, Croft \& Cruse [6] describes the notion of containment by explaining that finger is a meronym of hand, because finger is an original construal found in hand and essentially can be defined as part of hand. 
Following the same cognitive framework as suggested by Croft \& Cruse, meronymy can be defined as follows: if $\mathrm{A}$ is a meronym of $\mathrm{B}$ in a particular context, then any member a of the extension of $\mathrm{A}$ maps onto a specific member $\mathrm{b}$ of the extension of $\mathrm{B}$ of which it is constructed as a part, or it potentially stands in an intrinsically constructed relation of part to some actual or potential member of B.

Cruse uses the notion of lexical configuration in constructing a hierarchy, arguing that a lexical configuration is formed as a consequence of semantic relation between lexical items. Cruse further divides lexical configuration into three types, namely taxonomic hierarchies (hyponymy), part-whole hierarchies (meronymy), and non-branching hierarchies. A meronymy has its own form of hierarchy in constructing the structure of part-whole relation under the framework of Cruse's lexical configuration.

Each element of such hierarchy is arranged on a certain level, based on the relationship of each lexeme: whether a lexeme is directly related to the main domain, whether it needs other lexemes as an intermediary to be related with the main domain, and whether such a lexeme is not directly related but still connected in any way with the main domain. Here, the leveling follows the following rule: the first element occupies the 1st level, all elements directly related to the first element occupy the 2nd level, all elements directly related to the second element occupy the 3 rd level, and so on. For non-branching hierarchies, there is only one element on each level.

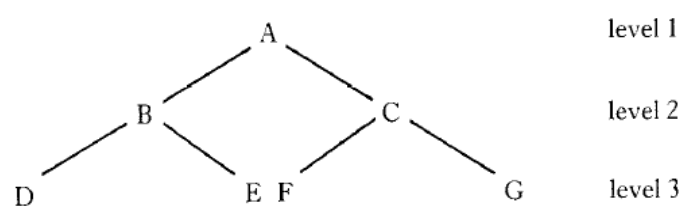

Fig1. Each Element of a Hierarchy on a Certain Level Cited from Cruse's

Hierarchies of part-whole relations of lexeme root cannot be properly analyzed by only visually observing the object in question. In this sense, corpus linguistics offers a more convenient methodology in lexical semantic studies by observing the occurrence frequency of lexeme root and its collocations. Corpus linguistics is a research approach that has been developed over the last few decades to support the empirical changes in language variation and language use, thus allowing more generalized findings with higher validity [7]. This claim is supported by Riemer [8] that states that corpora are very useful in semantic analyses as they can show unexpected collocational patterns or unusual word combinations, which are often overlooked.

\section{Research Method}

This study employs a corpus linguistic approach and uses the notion of lexical semantics in analyzing the object of study. In other words, this study integrates the qualitative method and the quantitative method into a mixed-methods approach. According to Greene [9], a mixedmethods approach is useful to produce a comprehensive finding and provide valid answers to the research questions. 
The data used was Wikipedia corpus that were taken from the BYU, an online corpora query system created by Davies [10]. With about 1.9 billion words taken from about 4.4 mission web pages, Wikipedia corpus (https://corpus.byu.edu/wiki) that was released in early 2015 has the richness and diversity to be used as research data. Before the hierarchal meronymy was constructed, the resulting collocates should be defined based on the descriptions provided by experts in plant botany such as Beck [11]; [12]; [13], in order to achieve scientifically valid and acceptable findings.

To construct the hierarchal meronymy of lexeme root, following Cruse's lexical configuration (1986). As previously mentioned, a lexical configuration is formed on each hierarchal level. Thus, Cruse's notion of lexical configuration does not adequately determine the parts of root meronymy on each hierarchal level. For that reason, this study integrates the corpus analysis that includes the collocation frequency, MI score, and concordance, and the scientific descriptions of parts of root as provided by experts in plant anatomy, in order to accurately determine which parts of root belong to each level of its meronymy hierarchy and to achieve a result that can be proven both linguistically and scientifically.

\section{Results And Discussion}

Root is the basest part of plant anatomy. It is the bottom part of a plant that grows in the soil and, therefore, is hard to observe. Nevertheless, root is the most important component of plants, especially those that live on land. The following is the number of lexeme root and its direct collocates found in Wikipedia Corpus.

Referring to the plant anatomy, a root is formed of several parts. As mentioned by Steeves and Sawhney, a root is formed of three tissue systems, namely dermal tissues, fundamental or ground tissues, vascular tissues and also root tip. Additionally, a root also has some outside part, namely bark.

\subsection{Root Bark}

The analysis begins from the outermost part of root, namely bark. The data of Wikipedia Corpus show a high frequency of occurrence of lexeme root that directly collocates with bark.

Table 1. The number of occurrence of lexeme root collocating with bark

\begin{tabular}{|c|c|c|c|c|c|}
\hline$\ddot{0}$ & Collocate & Frequency & Overall & Percentage & MI \\
\hline$\approx$ & bark & 236 & 17187 & 1.37 & 5.07 \\
\hline
\end{tabular}

Root is found to appear collocating with bark in 236 occurrences. The MI score of 5.07 shows a significantly close relationship between root and its meronyms. Further, the concordances result is as follows.

This bark beetle feeds on the developing bark on and around the root crowns of tree seedlings, especially the phloem with mycelial sheaths of the fungus beneath the bark of the root colla

The root bark and stem are analgesic, antiphlogistic, antirheumatic, hypoglycaemic and tonic

The part-whole relation in bark-root meronymy is marked by the prepositions on, of, and the prepositional adverb around. In the context, this bark beetle feeds on the developing bark on and around the root, the verb develop is found that denotes the meaning of bark growing around a root. This verb makes evident that bark is part of root that grows around it. These 
contexts found in the concordance of root collocating with bark indicate that bark is part of root and forms a meronymy with it.

\subsection{Root Epidermis}

Epidermic layers, or referred to by Steeves \& Sawhney as dermal tissues, are parts of root that come after bark. According to Steeves \& Sawhney, an epidermal cell has a thin layer of cuticle on the outside that thickens as the root ages. This definition can also explain bark, which is indeed a thick layer on the skin of older roots, resulting from epidermal cells in the epidermis. This affirms Cutler, et al. that mention that on all roots of plant, except those that grow on the soil or water, root hairs are usually found not far from the area where the roots grow, and they develop from rhizodermis or root epidermis.

The above descriptions are confirmed in the data, in which the words epidermal cells, cuticle, and root hairs, are found to collocate with the lexeme epidermis.

Table 2. The number of occurrence of lexeme epidermis and its collocates that denote parts of root

\begin{tabular}{rlllll}
\hline \multicolumn{1}{r}{ Collocate } & Frequency & Overall & Percentage & MI \\
\cline { 2 - 6 } & hair & 58 & 78771 & 0.07 & 5.62 \\
& root & 42 & 108537 & 0.04 & 4.69 \\
& cuticle & 39 & 1286 & 3.03 & 10.99 \\
& epidermal cell & 27 & 1252 & 2.16 & 10.49 \\
\hline
\end{tabular}

The resulting concordances, starting from root that collocates with epidermis, are as follows.

The cells of the root epidermis grow at a right angle to the axis of the root to allow them

In plants with secondary growth, the epidermis of roots and stems is usually replaced by a periderm

The part-whole relation between epidermis and root is marked by the preposition of and the verb grow. In the context, the cells of the root epidermis grow at a right angle to the axis of the root, the verb grow is found to denote the meaning of epidermal tissues growing on the root axis. This verb proves that epidermis is part of root that grows on the root tissues. These contexts found in the concordance of epidermis collocating with root indicate that epidermis is part of root and forms a meronymy with it. From this analysis, a branching hierarchy of meronymy can be constructed as follows.

\subsection{Root Cortex}

Cortex comes after epidermis as a part of root. Cortex, or referred to by Steeves \& Sawhney as a ground tissue, is located right below the epidermis. The deepest layer of cortex is a special cellular layer called the endodermis. These cells form suberin strips where fatty materials are deposited in the radial and transverse walls. These suberin strips are called the casparian strips Steeves \& Sawhney. In the data, the collocates of cortex are as found as follows:

Table 3. The number of occurrence of lexeme cortex and its collocates that denote parts of root

\begin{tabular}{|c|c|c|c|c|c|}
\hline \multirow{3}{*}{ ن⿺辶大 } & Collocate & Frequency & Overall & Percentage & MI \\
\hline & root & 37 & 10732 & 0.34 & 3.08 \\
\hline & endodermis & 13 & 10732 & 0.12 & 11.13 \\
\hline 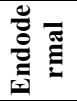 & casparian strip & 3 & 97 & 3.09 & 17.05 \\
\hline
\end{tabular}


The resulting concordances, starting from root that collocates with cortex, are as follows.

the cortex is the outermost layer of the stem or root of a plant, bounded on the outside by the epidermis They introduce necrosis in the cortex of the root which is detrimental to the plant

The part-whole relation between cortex and root is marked by the preposition of and the adjective outermost. The context, the cortex is the outermost layer of the stem or root of a plant denotes the meaning that cortex is located on the most outer side of root. The preposition of is also found in the context, the cortex of the root, denoting the meaning that cortex is part of root. These contexts found in the concordance of root collocating with cortex indicate that cortex is part of root and forms a meronymy with it.

\subsection{Root Stele}

After the ground tissues come the vascular tissues that are more known as stele. In some dicotyle plants, the middle part of the root may all be made of xylem, but in monocotyle plants and some other dicotyle plants the root, especially on the primary root, may contain some ground tissues that consist of parenchymas with a thin or thick wall called the pith. Eventually, xylem and phloem (and pith), with an addition of the pericycle, constitute the whole vascular tissues. In Wikipedia corpus, each of these parts of vascular tissues are found as follows.

Table 4. The number of occurrence of lexeme vascular tissue and its collocates that denote parts of root

\begin{tabular}{llllll}
\hline \multicolumn{1}{c}{ Collocate } & Frequency & Overall & Percentage & MI \\
\cline { 2 - 6 } & root & 11 & 108537 & 0.01 & 5.75 \\
& 5 & 504 & 0.99 & 12.36 \\
& xylem & 3 & 415 & 0.72 & 11.90 \\
& phloem & 1 & 24 & 4.17 & 14.43 \\
& pericycle & 1 & 643 & 0.16 & 9.68 \\
\hline
\end{tabular}

The resulting concordances, starting from root that collocates with vascular tissue, are as follows.

This results in discolored or necrotic vascular tissue in the root, and the tissue bordering

root hair, epidermis, epiblem, cortex, endodermis, pericycle and lastly the vascular tissue in the centre of a root responsible for most water transport through the vascular tissue in stems and roots

The part-whole relation between vascular tissue and root is marked by the preposition in and the noun centre. In the context, most water transport through the vascular tissue in stems and roots, the preposition in creates the meaning that vasculat tissue is located inside the root. These contexts found in the concordance of root collocating with vascular tissue indicate that vascular tissue is part of root and forms a meronymy with it.

\subsection{Root Tip}

The tip of root is called root apex, or simply known as root tip. There are several parts of the root tip, namely root cap, quiescent center, protoderm, and meristem. In Wikipedia corpus, the following data are found.

Table 5. The number of lexeme root and its collocates regarding root tip.

\begin{tabular}{|c|c|c|c|c|c|}
\hline \multirow{4}{*}{$\begin{array}{l}\ddot{\circ} \\
\stackrel{0}{\mathscr{u}}\end{array}$} & Collocate & Frequency & Overall & Percentage & MI \\
\hline & root tip & 55 & 108537 & 0.05 & 10.24 \\
\hline & root cap & 5 & 108537 & 0.00 & 7.78 \\
\hline & quiescent center & 1 & 108537 & 0.00 & 7.40 \\
\hline
\end{tabular}




\begin{tabular}{|c|c|c|c|c|c|}
\hline$\simeq 8-$ & Collocate & Frequency & Overall & Percentage & $\overline{\mathrm{MI}}$ \\
\hline$\vec{\circ}: \vec{\Xi}$ & meristem & 1 & 8 & 12.50 & 18.19 \\
\hline 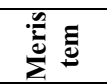 & protoderm & 1 & 372 & 0.27 & 16.53 \\
\hline
\end{tabular}

The lexeme root directly collocate with root tip, root cap, and quiescent center. In the data, although protoderm is not found to directly collocate with root tip, it is found to collocate with mersitem. This conforms the description by the experts in plant anatomy that protoderm is the first layer on the meristem. Meanwhile, the resulting concordances, starting from root apex or root tip that collocates with root, are as follows.

Removal of the root tip can lead to inhibition of secondary root formation

tissues of the root, first undergoing elongation, a process that pushes the root tip forward in the growing medium release zoospores, which infect plant roots by entering the root behind the root tip

The part-whole relation between root tip and rootis marked by the prepositional adverb behind. In the context, plant roots by entering the root behind the root tip, the adverb behind denotes the location where the root tip is located on the root. This context indicates that root tip is part of root and forms a meronymy with it.

From the analysis performed on each co-meronymy of lexeme root in relation with plant, a hierarchy of root meronymy can be formed from the combination of each part of the root as follows.

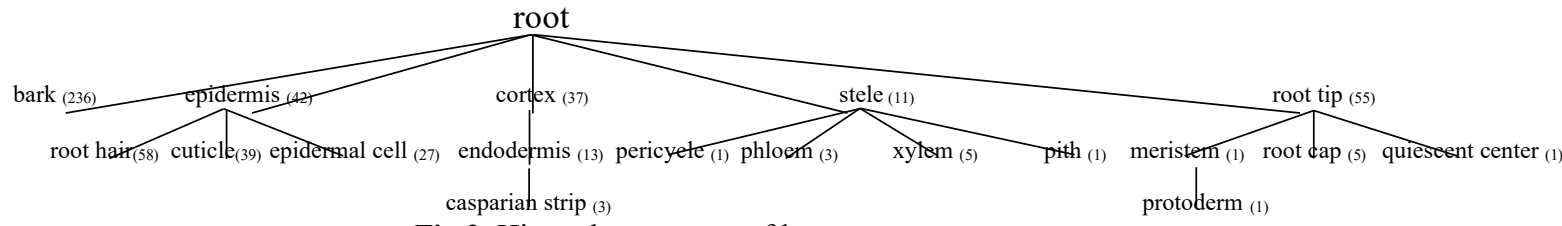

Fig 2. Hierarchy structure of lexeme root

\section{Conclusion}

As made evident from the BYU, root is a dominant part of a plant. In the root hierarchy, the second level is occupied by lexeme bark, epidermis, cortex, stele or vascular tissue, and root tip or root apex. The third level of the hierarchy is occupied by root hair, cuticle, epidermal cell, endodermis, pericycle, phloem, xylem, pith, meristem, root cap, and quiescent center. Finally, the forth level is occupied by casparian strip and prototerm. Overall, the hierarchy formed on each lexeme of root consists of four levels, the arrangement of which is based on the descriptions made by experts in plant botany and the data in Wikipedia Corpus.

In line with the characteristics of Cruse's meronymy, the hierarchy constructed in this study contains the same elements related to the lexeme root and directly or indirectly collocating with it. For instance, lexeme xylem and phloem are both part of root but do not directly collocate with root. Instead, both are related to root by directly collocating with stele or vascular tissue, based on the descriptions provided by experts in plant anatomy. This shows that not all lexeme related to root in Wikipedia corpus are found to directly collocate with it. 


\section{References}

[1] M. L. Murphy, Lexical Meaning. Cambridge: Cambridge University Press, 2010.

[2] M. L. Murphy, Semantic Relations and the Lexicon: Antonymy, Synonymy and other Paradigms. Cambridge: Cambridge University Press, 2003.

[3] D. A. Cruse, "On the Transitivity of the Part-Whole Relation," Journal of Linguistics, vol. 15, pp. 29-38. Cambridge: Cambridge University Press, 1979.

[4] D. A. Cruse, Meaning in Language: An Introduction to Semantics and Pragmatics. United States of America: Oxford University Press, 2000.

[5] W. Croft \& D. A. Cruse, Cognitive Linguistics. Cambridge: Cambridge University Press, 2004.

[6] D. A. Cruse, Lexical Semantics. Cambridge: Cambridge University Press, 1986.

[7] D. Biber, "Corpus-Based and Corpus-Driven Analyses of Language Variation and Use," in B. Heine \& H. Harrog, Eds, The Oxford Handbook of Linguistics Analysis. Oxford: Oxford University Press, pp. 159-191, 2010.

[8] N. Riemer, Introducing Semantics. Cambridge: Cambridge University Press, 2010.

[9] J. C. Greene, V. J. Caracelli, \& W. F. Graham, "Toward a Conceptual Framework for MixedMethod Evaluation Designs," Educational Evaluation and Policy Analysis, vol. 11, pp. 255-274, 1989.

[10] M. Davies, “The Wikipedia Corpus," January, 2015. [Online]. Available: https://corpus.byu.edu/wiki.

[11] C. B. Beck, An Introduction to Plant Structure and Development: Plant Anatomy for the Twenty-First Century. United States of America: Cambridge University Press, 2010.

[12] D. F. Cutler, T. Botha, \& D. W. Stevenson, Plant Anatomy: An Applied Approach. United Kingdom: Blackwell Publishing Ltd., 2007.

[13] T. A. Steeves \& V. K. Sawhney, Essentials of Developmental Plant Anatomy. States of America: Oxford University Press, 2017. 